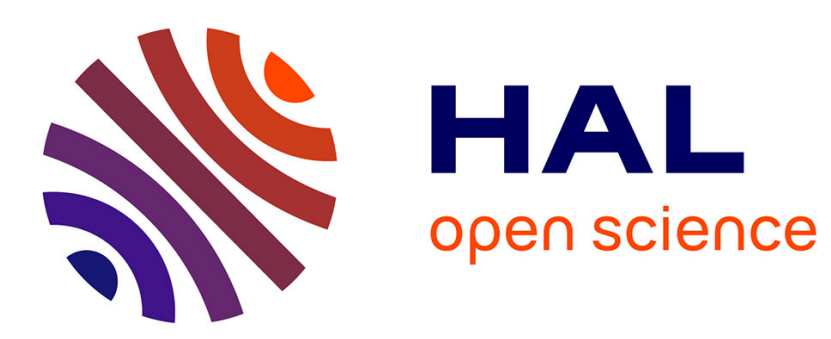

\title{
Completion in Operads via Essential Syzygies
}

Philippe Malbos, Isaac Ren

\section{To cite this version:}

Philippe Malbos, Isaac Ren. Completion in Operads via Essential Syzygies. 46th International Symposium on Symbolic and Algebraic Computation, Jul 2021, Saint Petersburg, Russia. 10.1145/3452143.3465552 . hal-03258883

\section{HAL Id: hal-03258883 \\ https://hal.science/hal-03258883}

Submitted on 12 Jun 2021

HAL is a multi-disciplinary open access archive for the deposit and dissemination of scientific research documents, whether they are published or not. The documents may come from teaching and research institutions in France or abroad, or from public or private research centers.
L'archive ouverte pluridisciplinaire HAL, est destinée au dépôt et à la diffusion de documents scientifiques de niveau recherche, publiés ou non, émanant des établissements d'enseignement et de recherche français ou étrangers, des laboratoires publics ou privés. 


\section{Completion in Operads via Essential Syzygies}

\author{
Philippe Malbos \\ Univ Lyon, Université Claude Bernard Lyon 1 \\ CNRS UMR 5208, Institut Camille Jordan \\ France
}

\begin{abstract}
We introduce an improved Gröbner basis completion algorithm for operads. To this end, we define operadic rewriting systems as a machinery to rewrite in operads, whose rewriting rules do not necessarily depend on an ambient monomial order. A Gröbner basis of an operadic ideal can be seen as a confluent and terminating operadic rewriting system; thus, the completion of a Gröbner basis is equivalent to the completion of a rewriting system. We improve the completion algorithm by filtering out redundant S-polynomials and testing only essential ones. Finally, we show how the notion of essential S-polynomials can be used to compute Gröbner bases for syzygy bimodules. This work is motivated by the computation of minimal models of associative algebras and symmetric operads. In this direction, we show how our completion algorithm extends to the case of shuffle operads.
\end{abstract}

\section{CCS Concepts}

- Computing methodologies $\rightarrow$ Combinatorial algorithms; Algebraic algorithms.

\section{Keywords}

Non commutative Gröbner bases; computing with syzygies; completion algorithms; rewriting in operads

\section{Introduction}

The fundamental theorem of any algebraic rewriting machinery is the critical branching theorem, or Buchberger criterion, which characterizes confluence by algebraic obstructions described as critical branchings or critical pairs in rewriting theory $[14,20]$, and as $S$-polynomials in Gröbner basis theory $[4,19]$. This theorem is the core of completion algorithms of a rewriting system, which resolve the obstructions in order to reach confluence. The efficiency of completion algorithms depends on several aspects: 1/ the algebraic structure on which we rewrite (strings, commutative or non-commutative polynomials, planar trees...), 2/ the strategy used to orient the rules added during completion, 3 / the choice of which critical branchings to resolve and the prediction non-essential ones, 4/ the order in which we resolve these critical branchings. For instance, Buchberger's algorithm consists in exploring all branchings, but several methods have been introduced to improve completion by reducing the set to be explored via syzygies of generating polynomials, which describe relations between obstructions $[9,15]$.

The objective of this work is twofold. Firstly, we introduce a rewriting machinery for non-symmetric operads that generalizes the Gröbner basis approach of $[3,8]$ by removing the constraint of a monomial order for the orientation of the rewriting rules. Secondly, we define a completion algorithm for rewriting in an operadic context by restricting the examination of critical branchings to essential

\author{
Isaac Ren \\ École Normale Supérieure de Lyon \\ France
}

ones. The essential branchings are defined by considering relations between critical branchings with respect to a monomial order on contexts. We show that the relations between these branchings correspond to syzygies of the defining rewriting rules.

This work is aimed at developing rewriting methods for calculating with operads. Operads provide an algebraic language to describe types of algebras in topology and algebra [16, 18]. They are usually defined with symmetric actions, which are incompatible with rewriting, but shuffle operads, introduced by Dotsenko and Khoroshkin in [7], allow for rewriting methods all while preserving the information of symmetries. In particular, these rewriting methods apply to the computation of explicit free resolutions of operads [7, 17]. The problem of finding a procedure that computes a minimal model for a given operad is still open. In the rewriting approach, this calls for a notion of minimal branchings in all dimensions of the resolution. In this article, we present our algorithm in the case of non-symmetric operads, but the shuffle case can be treated in the same way. Moreover, our notion of essential branchings can also be used to 'go up in dimension', and we expect to deduce an algorithm calculating minimal resolutions of operads.

The paper is structured as follows. In Section 2, we define the notions of rewriting systems and Gröbner bases for non-symmetric operads. We introduce the notion of essential branchings, and we give an algorithm to compute essential branchings with respect to a monomial order on contexts. In Section 3, we show the main result of this paper, Theorem 3.3, which characterizes the confluence of a terminating operadic rewriting system in terms of essential branchings. From this result, we deduce a completion algorithm, which transforms terminating rewriting systems into confluent ones by resolving essential branchings. In Section 4, we show that essential syzygies generate the bimodule of all syzygies of a convergent operadic rewriting system. As a conclusion, in Section 5, we give an alternative definition of essential branchings, allowing us to define essential branchings involving $n>2$ rewriting rules. We also explain how to adapt our constructions to the cases of algebras over operads and shuffle operads.

\section{Operadic rewriting systems}

In this section, we introduce rewriting systems for non-symmetric operads, which we relate to the notion of Gröbner bases for operads. We define the notion of essential branchings as 'minimal' obstructions to local confluence, and an algorithm to compute the essential branchings of an operadic rewriting system.

Operads. A collection is a sequence $V=(V(n))_{n \in \mathbb{N}}$ of spaces (over a field $\mathbf{k}$ ) indexed by arities $n \geqslant 0$. We denote by $\operatorname{AR}(x)$ the arity of an element of $V$. A (non-symmetric) operad is a collection $P$ with an identity element $\varepsilon$ in $P(1)$, equipped with composition maps

$$
\circ: P(k) \otimes P\left(n_{1}\right) \otimes \ldots \otimes P\left(n_{k}\right) \rightarrow P\left(n_{1}+\ldots+n_{k}\right)
$$


sending $\left(x, y_{1}, \ldots, y_{k}\right)$ to $x \circ\left(y_{1}, \ldots, y_{k}\right)$ and satisfying identity and associativity conditions:

$$
\text { i) } \begin{aligned}
& x \circ(\varepsilon, \ldots, \varepsilon)=x=\varepsilon \circ x \\
\text { ii) } & x \circ\left(y_{1} \circ\left(z_{1}^{1}, \ldots, z_{k_{1}}^{1}\right), \ldots, y_{j} \circ\left(z_{1}^{j}, \ldots, z_{k_{j}}^{j}\right)\right) \\
& \left.=\left(x \circ\left(y_{1}, \ldots, y_{j}\right)\right) \circ\left(z_{1}^{1}, \ldots, z_{k_{1}}^{1}, \ldots, z_{1}^{j}, \ldots, z_{k_{j}}^{j}\right)\right)
\end{aligned}
$$

For $1 \leqslant i \leqslant k, x \in P(k)$, and $y \in P(n)$, denote by $x \circ_{i} y:=$ $x \circ(\varepsilon, \ldots, \varepsilon, y, \varepsilon, \ldots, \varepsilon)$ an elementary composition of $x$ and $y$. $i$

The set of (tree) monomials $\mathcal{T}(\Sigma)$ is the term algebra on a graded set $\Sigma=\left(\Sigma_{n}\right)_{n \geqslant 0}$. Monomials will be written using the Backus-Naur form

$$
\mathcal{T}(\Sigma)::=\{\varepsilon\} \mid\left(\Sigma(k) \mid \mathcal{T}(\Sigma)\left(n_{1}\right) \ldots \mathcal{T}(\Sigma)\left(n_{k}\right)\right) .
$$

We denote by $\vec{v}$ the list of monomials $v_{1}, \ldots, v_{k}$. A monomial can be represented by a planar tree with inputs: for instance,

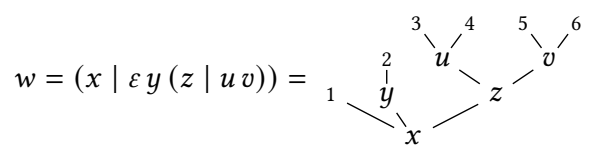

is a monomial where $\operatorname{AR}(x)=3, \operatorname{AR}(y)=1$, and $\operatorname{AR}(z)=\operatorname{AR}(u)=$ $\operatorname{AR}(v)=2$. The size of a monomial $u$ is the number of inner vertices. For instance, $\operatorname{sizE}(w)=5$.

In this work, we rewrite in free operads. We denote by $\mathcal{F}(\Sigma)$ the free operad over $\Sigma$, where, for $n>0, \mathcal{F}(\Sigma)(n)$ is the vector space spanned by monomials of arity $n$, whose elements are called (homogeneous) polynomials. The support of $f=\sum_{i \in I} \lambda_{i} u_{i}$ is the set of monomials $\operatorname{Supp}(f):=\left\{u_{i} \mid i \in I\right\}$ that appear in its decomposition. The elementary compositions of monomials extend to polynomials by multilinearity.

A (one-hole) monomial context of $\mathcal{F}(\Sigma)$ of inner arity $k$ is a term $C:=w \circ_{i}\left(\square_{k} \mid \vec{w}\right)$ of $\mathcal{T}\left(\Sigma \cup\left\{\square_{k}\right\}\right)$, where $\square_{k}$ is a symbol of arity $k$ that appears exactly once in $C$. For a polynomial $f$ in $\mathcal{F}(\Sigma)(k)$, we denote by $C[f]$ the polynomial $w \circ_{i}(f \mid \vec{w})$. A (one-hole) context of $\mathcal{F}(\Sigma)$ of (inner) arity $k$ is a linear combination of one-hole monomial context of inner arity $k$. Similarly, we define a two-hole context of $\mathcal{F}(\Sigma)$ inner arities $(k, \ell)$ as a linear combination of terms of $\mathcal{T}\left(\Sigma \cup\left\{\square_{k}\right\} \cup\left\{\square_{\ell}\right\}\right)$ with exactly one occurence of both $\square_{k}$ and $\square_{\ell}$. When the arity of $D$ (as a monomial) is equal to the inner arity of $C$, we can compose the two contexts, which we denote by $C D$. Finally, for monomials $u, v$, we write $u \subseteq v$ when there exists a monomial context $C$ such that $v=C[u]$.

An $\mathcal{F}(\Sigma)$-bimodule $M$ is a collection $M$ equipped with an action of the collection of contexts of $\mathcal{F}(\Sigma)$ satisfying compatibility axioms with respect to the composition of $\mathcal{F}(\Sigma)$. An ideal of $\mathcal{F}(\Sigma)$ is an $\mathcal{F}(\Sigma)$-bimodule contained in $\mathcal{F}(\Sigma)$. Given a family of polynomials $F=\left\{f_{1}, \ldots, f_{s}\right\} \subseteq \mathcal{F}(\Sigma)$, we denote by $I(F)$ the ideal generated by $F$ and by $\mathcal{F}(\Sigma)\langle F\rangle$ the free $\mathcal{F}(\Sigma)$-bimodule generated by $F$. The $\mathcal{F}(\Sigma)$-bimodule of syzygies of $F$, denoted by $\mathcal{S}(F)$, is the kernel of the bimodule morphism $\mathcal{F}(\Sigma)\langle F\rangle \rightarrow \mathcal{F}(\Sigma)$. Thus, a syzygy corresponds to a relation

$$
C_{1}\left[f_{1}\right]+\ldots+C_{s}\left[f_{s}\right]=0,
$$

where the $C_{i}$ are polynomial contexts of $\mathcal{F}(\Sigma)$.

Operadic rewriting systems. An operadic rewriting system, or ORS, is a data $X=(\Sigma, R)$ made of a graded set $\Sigma$ and a (binary) relation $R \subset \mathcal{T}(\Sigma) \times \mathcal{F}(\Sigma)$, whose elements are rules $\alpha: u \rightarrow f$ reducing the monomial source $s(\alpha):=u$ to the polynomial target $t(\alpha):=f$. We write $\partial=s-t$ and define the ideal generated by $R$ as $I(R):=\langle\partial(\alpha) \mid \alpha \in R\rangle$. The operad presented by the ORS $X$, denoted by $\bar{X}$, is the quotient of the free operad $\mathcal{F}(\Sigma)$ by $I(R)$.

We define the graph $\mathcal{R}_{X}$, whose vertices are the elements of $\mathcal{F}(\Sigma)$ and whose edges are the $\lambda C[\alpha]+1_{g}: \lambda C[s(\alpha)]+g \rightarrow_{R}$ $\lambda C[t(\alpha)]+g$, where $\alpha \in R, C$ is a monomial context, $\lambda \in \mathbf{k} \backslash\{0\}$, and $g$ is a polynomial of $\mathcal{F}(\Sigma)$. For every edge $a$ of $\mathcal{R}_{X}$, denote by $a^{-}=s(a)-a+t(a)$ the edge with source $t(a)$ and target $s(a)$. An edge of $\mathcal{R}_{X}$ is a rewriting monomial when $\lambda=1$ and $g=0$, and a rewriting step when $C[s(\alpha)] \notin \operatorname{Supp}(g)$. Denote by $\mathcal{R}_{X}^{m}\left(\operatorname{resp} . \mathcal{R}_{X}^{+}\right)$ the set of rewriting monomials (resp. rewriting steps) of $X$. Denote by - the composition of paths in $\mathcal{R}_{X}$. A composition of rewriting steps is called a rewriting path of $X$. A polynomial $f$ in $\mathcal{F}(\Sigma)$ is in normal form wrt $X$, or reduced, if there is no rewriting step with source $f$. Denote by $\mathrm{NF}(X)$ the set of reduced polynomials of $\mathcal{F}(\Sigma)$.

An order relation $<$ on $\mathcal{T}(\Sigma)$ is compatible with $R$ if, for every $\alpha \in R$ and every monomial $v \in \operatorname{Supp}(t(\alpha))$, we have $v<s(\alpha)$. The relation $\prec$ extends to $\mathcal{F}(\Sigma)$ by setting, for $f, g$ in $\mathcal{F}(\Sigma), g \prec f$ if the two following conditions are satisfied:

i) $\operatorname{Supp}(f) \backslash \operatorname{Supp}(g) \neq \varnothing$,

ii) for all $v \in \operatorname{Supp}(g) \backslash \operatorname{Supp}(f)$, there exists $u \in \operatorname{Supp}(f) \backslash$ $\operatorname{Supp}(g)$ such that $v<u$.

We denote by $\prec_{R}$ the smallest partial order relation on $\mathcal{T}(\Sigma)$ stable by product and compatible with $R$. The ORS $X$ is terminating if the relation $\prec_{R}$ is well-founded. In this case, there does not exist infinite sequence of rewriting steps of $X$.

Rewriting monomials form a basis of $\mathcal{F}(\Sigma)\langle R\rangle$, the free $\mathcal{F}(\Sigma)$ bimodule on $R$, whose elements we call rewriting polynomials. A syzygy then corresponds to a rewriting polynomial $\mathfrak{s}$ such that $\partial(\mathfrak{s})=0$. We denote by $\mathcal{S}(X)$ the $\mathcal{F}(\Sigma)$-bimodule of syzygies of $X$, which corresponds to the bimodule $\mathcal{S}(\partial(R))$ defined previously.

Monomial orders. A monomial order on $\mathcal{T}(\Sigma)$ is a total order $\prec$ stable by product; that is, for all $u, u^{\prime} \in \mathcal{T}(\Sigma)(k), v, v^{\prime} \in \mathcal{T}(\Sigma)(\ell)$, and $1 \leqslant i \leqslant k, u<u^{\prime}, v<v^{\prime}$ implies $u \circ_{i} v<u^{\prime} \circ_{i} v^{\prime}$. A monomial order on $\mathcal{T}\left(\Sigma \sqcup\left\{\square_{k}\right\}_{k \geqslant 1}\right)$ induces a monomial order on contexts of $\mathcal{F}(\Sigma)$. Given a monomial order $\prec$ on $\mathcal{T}(\Sigma)$, a monomial order on contexts, and a total order $<$ on $R$, we define the rewriting monomial order $\prec_{\text {rm }}$ on $\mathcal{R}_{X}^{m}$ by setting $C[\alpha] \prec_{\text {rm }} D[\beta]$ if
i) $C[s(\alpha)]<D[s(\beta)]$, or
ii) $C[s(\alpha)]=D[s(\beta)]$ and $C<D$, or
iii) $C[s(\alpha)]=D[s(\beta)], C=D$, and $\alpha<\beta$.

Note that the third case only occurs if the ORS admits rules with the same source.

An example of a monomial order is the path-lexicographic order, defined in $[6,12]$. Given a tree monomial, there exists a unique path from the root to each input. We can write these paths as words in the alphabet $\Sigma$ : for instance, the monomial $w=(x \mid \varepsilon y(z \mid u v))$ above gives the paths $(x, x y, x z u, x z u, x z v, x z v)$. Given a total order $\prec$ on $\Sigma$, we define the lexicographic order $\prec_{\text {lex }}$ on paths. Then, we define the path-lexicographic order $\prec_{\text {path-lex }}$ as the lexicographic product order of $\prec_{\text {lex }}$ on the list of paths, ordered by the inputs.

We can extend $\prec$ to a total order $\prec^{\prime}$ on $\Sigma \sqcup\left\{\square_{k}\right\}_{k \geqslant 1}$ where, for all $1 \leqslant k<\ell$ and $x \in \sum, \square_{k} \prec^{\prime} \square_{\ell}$ and $\square_{k} \prec^{\prime} x$. We can then define the path-lexicographic order $\prec_{\text {path-lex }}^{\prime}$ on $\mathcal{T}\left(\Sigma \sqcup\left\{\square_{k}\right\}_{k}\right)$, which 
restricts to a monomial order $\prec_{\text {cont-path-lex }}$ on monomial contexts. Fixing a total order on $R$, this gives a rewriting monomial order $\prec_{\text {rm-path-lex }}$.

Gröbner bases. Fix a monomial order $\prec$ on $\mathcal{T}(\Sigma)$. The leading monomial of a polynomial $f$ of $\mathcal{F}(\Sigma)$ is the greatest monomial in the support of $f$ wrt the order $\prec$, denoted by $\operatorname{lm}_{<}(f)$. We define the associated rule $\overrightarrow{\operatorname{lm}}_{<}(f): \operatorname{lm}_{<}(f) \rightarrow 1 / \mathrm{lc}_{<}(f)\left(f-\operatorname{lm}_{<}(f)\right)$, where $\mathrm{lc}_{<}(f)$ denotes the coefficient of $\operatorname{lm}_{<}(f)$ in the polynomial $f$. For a set of polynomials $G$ of $\mathcal{F}(X)$, we denote by $\overrightarrow{\operatorname{lm}}_{<}(G)$ := $\left\{\overrightarrow{\mathrm{m}}_{<}(g) \mid g \in G\right\}$ the corresponding set of rules. We say that $G$ is a Gröbner basis of an ideal $I$ of $\mathcal{F}(\Sigma)$ if $G$ generates $I$ and the ORS $\left(X, \overrightarrow{\operatorname{lm}}_{<}(G)\right)$ is confluent, or equivalently, if every polynomial $f$ in $I$ reduces to 0 wrt $\overrightarrow{\operatorname{lm}}_{<}(G)$.

Now, let $E$ be a set of rewriting polynomials. Every element e of $E$ can be written $\mathrm{e}=\sum_{i} \lambda_{i} C_{i}\left[\alpha_{i}\right]$, where $\lambda_{i} \in \mathbf{k} \backslash\{0\}, C_{i}$ is a monomial context, and $\alpha_{i} \in R$. We define the rule $\overrightarrow{l m}_{<_{\mathrm{rm}}}(\mathfrak{e}): \operatorname{lm}_{\succ_{\mathrm{rm}}}(\mathfrak{e}) \rightarrow$ $1 / \mathrm{lc}_{<_{\mathrm{rm}}}(\mathfrak{e})\left(\mathfrak{e}-\operatorname{lm}_{<_{\mathrm{rm}}}(\mathfrak{e})\right)$, where $\mathrm{lc}_{<_{\mathrm{rm}}}(\mathfrak{e})$ denotes the coefficient of $\operatorname{lm}_{\prec_{\mathrm{rm}}}(\mathfrak{e})$ in e. Denote by $\overrightarrow{\mathrm{lm}}_{\succ_{\mathrm{rm}}}(E)$ the set of these rules for $\mathfrak{e} \in E$. We say that $E$ is a Gröbner basis of a submodule $M$ of $\mathcal{F}(\Sigma)\langle R\rangle$ wrt $\prec_{\text {rm }}$ if $E$ generates $M$ as a $\mathcal{F}(\Sigma)$-bimodule and every rewriting polynomial $\mathfrak{s}$ in $M$ reduces to 0 wrt $\overrightarrow{\mathrm{m}}_{\prec_{\mathrm{rm}}}(E)$.

Branchings and confluence. A branching (resp. local branching) is a pair $(a, b)$ of rewriting paths (resp. rewriting steps) such that $a \neq b$ and $s(a)=s(b)$. We classify local branchings into three types:

i) additive branchings: $\left(\lambda a+\mu 1_{v}+1_{h}, \lambda 1_{u}+\mu b+1_{h}\right)$, where $a: u \rightarrow f, b: v \rightarrow g \in \mathcal{R}_{X}^{m}, \lambda, \mu \in \mathbf{k} \backslash\{0\}, h$ is a polynomial, $u \neq v$, and $u, v \notin \operatorname{Supp}(h)$.

ii) multiplicative branchings: $\left(\lambda C\left[a, 1_{v}\right]+1_{h}, \lambda C\left[1_{u}, b\right]+1_{h}\right)$, where $C$ is a monomial two-hole context, $a: u \rightarrow f, b:$ $v \rightarrow c \in \mathcal{R}_{X}^{m}, \lambda \in \mathbf{k} \backslash\{0\}, h$ is a polynomial, and $C[u, v] \notin$ $\operatorname{Supp}(h)$.

iii) intersecting branchings: the rest of the local branchings.

We define a well-founded partial order $\sqsubseteq$ on branchings by setting, for every monomial context $C$, polynomial $h$, and $\lambda \in \mathbf{k} \backslash\{0\}$,

$$
(a, b) \sqsubseteq\left(\lambda C[a]+1_{h}, \lambda C[b]+1_{h}\right) .
$$

The critical branchings are the minimal intersecting branchings for this order.

Now, fix a rewriting monomial order $\prec_{\mathrm{rm}}$ on $\mathcal{R}_{X}^{m}$. An essential branching of $X$ is a critical branching $(C[\alpha], D[\beta])$, where $C[\alpha] \prec_{\mathrm{rm}} D[\beta]$ and they are consecutive for this order, that is there does not exist a rewriting monomial $E[\gamma]$ such that $C[\alpha] \prec_{\text {rm }}$ $E[\gamma] \prec_{\text {rm }} D[\beta]$. We denote by $\mathcal{E}(X)$ the set of essential branchings of $X$. Algorithm 1 computes the essential branchings of an ORS $X$ with respect to a monomial order.

The S-polynomial of a branching $(a, b)$ of $X$ is the polynomial $S(a, b):=t(a)-t(b)$. It is called essential when the branching is so. A branching $(a, b)$ is confluent if there exist two rewriting paths $t(a) \rightarrow h$ and $t(b) \rightarrow h$ with the same target, or equivalently, if its $S$-polynomial reduces to 0 . The ORS $X$ is confluent (resp. locally confluent) at a polynomial $f$ if all its branchings (resp. local branchings) with source $f$ are confluent. It is confluent (resp. locally confluent) if it is so at every polynomial, and convergent if it is confluent and terminating.

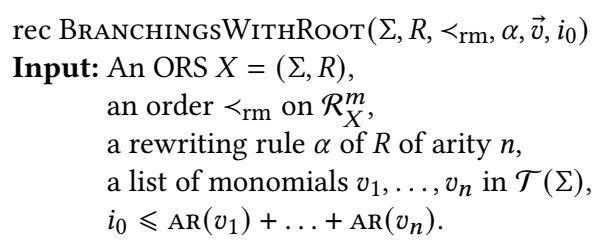

Output: A set of essential branchings whose first rewriting step contains $(\alpha \mid \vec{v})$, and where the first $i_{0}-1$ inputs are untouched.

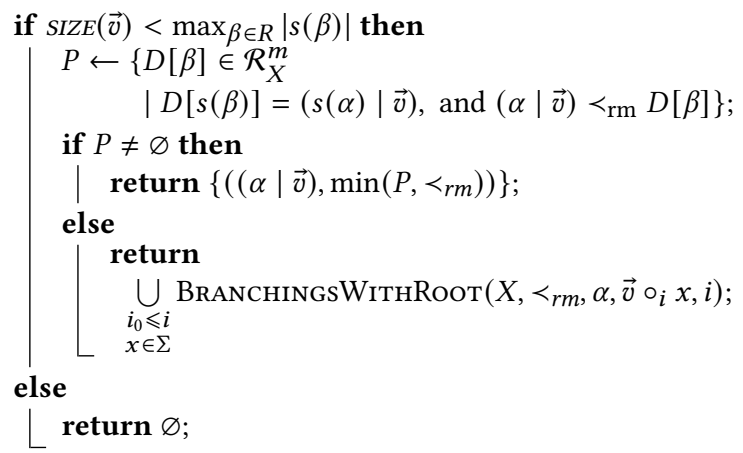

EsSENTIALBRANCHINGS $\left(\sum, R, \prec_{\text {rm }}\right)$

Input: An ORS $X=(\Sigma, R)$,

A rewriting monomial order $\prec_{\text {rm }}$ on $\mathcal{R}_{X}^{m}$.

Output: The set of essential branchings of $(\Sigma, R)$.

return $\bigcup_{\alpha \in R} \operatorname{BranchingsWithRoOT}\left(\Sigma, R, \prec_{r m}, \alpha, \vec{\varepsilon}, 1\right)$;

Algorithm 1: EssentialBranchings and its auxiliary recursive function, BRANCHINGSWITHROOT

Let $X$ be a convergent ORS, $<$ a monomial order on $\mathcal{T}(\Sigma)$, and $<$ a total order on $R$, and consider the associated monomial order $<_{\text {rm }}$ on rewriting monomials. A normalization strategy $\rho$ associates to each polynomial $f$ of $\mathcal{F}(X)$ a rewriting path from $f$ to its normal form as follows. If $f$ is a normal form, then $\rho_{f}:=1_{f}$. Otherwise, write $f=\lambda u+g$, where $u$ is the greatest reducible monomial of $f$ wrt $\prec, \lambda \in \mathbf{k} \backslash\{0\}$, and $u \notin \operatorname{Supp}(g)$, and let $C[\alpha]$ be the greatest rewriting monomial wrt $\prec_{\text {rm }}$ that reduces $u$. We then set

$$
\rho_{f}:=(\lambda C[\alpha]+g) \cdot \rho_{\lambda C[t(\alpha)]+g}
$$

Since $X$ is terminating, $\rho$ is well-defined.

Examples.

i) Consider the associative operad presented by one generator $x$ and one rule:

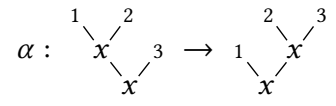

also written $x \circ_{1} x \rightarrow x \circ_{2} x$. There is one critical branching, and so one essential branching, $\left(\alpha \circ_{1} x, x \circ_{1} \alpha\right)$.

ii) Consider the associative algebra presented by $\langle x| \alpha$ : $\left.x^{3} \rightarrow 0\right\rangle$, seen as an operad concentrated in arity 1 . The critical branchings are $(\alpha x, x \alpha)$ and $(x x \alpha, \alpha x x)$, and only the first one is essential. 
iii) Consider the ORS with three generators $x, y, z$ and the following rewriting rule:

$$
(x \mid y z) \rightarrow(x \mid x x)+(y \mid y y)+(z \mid z z)
$$

There are no essential branchings. However, this rule cannot be the result of an orientation by a monomial order, since there is always a monomial on the righthand side greater than $(x \mid y z)$. Note that if we orient this rule by a monomial order, we would get two essential branchings.

\section{Completion using essential branchings}

In this section, we show that the obstructions to the confluence of a terminating ORS can be reduced to the confluence of the essential branchings, which, as we will explain in the next section, corresponds to existence of essential syzygies. We deduce an improved completion algorithm for operadic rewriting systems.

LeMma 3.1 ([11]). Let $X$ be an ORS.

i) For every path a in $\mathcal{R}_{X}$, there exists a zig-zag sequence of rewriting steps from $s(a)$ to $t(a)$.

ii) For every path $f_{0} \stackrel{a_{1}}{\longrightarrow} f_{1} \stackrel{a_{2}}{\longrightarrow} \ldots \stackrel{a_{n}}{\longrightarrow} f_{n}$ of length $n$ such that $X$ is confluent at $f_{i}$ for all $i \geqslant 1$, there exist rewriting paths $b$ and $c$ such that $b \cdot c^{-}$has source $f_{0}$ and target $f_{n}$.

Proof. Consider an edge $a=\lambda C[\alpha]+1_{g}$ of $\mathcal{R}_{X}$. Write $g=$ $\mu C[s(\alpha)]+h$, where $C[s(\alpha)] \notin \operatorname{Supp}(h)$ and $\mu \in \mathbf{k}$. Then the zigzag of rewriting paths

$$
\left((\lambda+\mu) C[\alpha]+1_{h}\right) \cdot\left(\mu C[\alpha]+1_{\lambda C[t(\alpha)]+h}\right)^{-}
$$

has the same source and target as $a$. We deduce the first point immediately.

For the second point, we proceed by induction on the length $n$ of the path. We have proven the point for $n=1$. For $n \geqslant 1$, consider the diagram

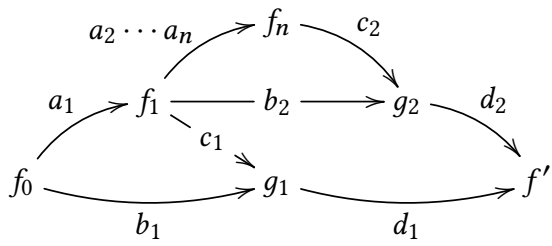

The rewriting paths $b_{1}, c_{1}$ are given by the first point, and the rewriting paths $b_{2}, c_{2}$ are given by induction hypothesis. Since we suppose $X$ confluent at $f_{1}$, we get the rewriting paths $d_{1}, d_{2}$. Thus $\left(b_{1} \cdot d_{1}\right) \cdot\left(c_{2} \cdot d_{2}\right)^{-}$has source $f_{0}$ and target $f_{n}$.

Lemma 3.2. Let $X$ be an ORS, $f$ a polynomial such that $X$ is confluent at every polynomial $g<_{R} f$, and $(a, b)$ a local branching of source $f$. Then $(a, b)$ is confluent if there exist rewriting paths $c, d$, one-hole contexts $C, D$ of $\mathcal{F}(\Sigma)$, and polynomials $h, k$ in $\mathcal{F}(\Sigma)$ as in the following diagram

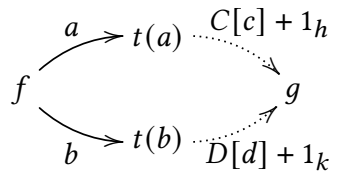

Proof. We shall construct the following confluence diagram:

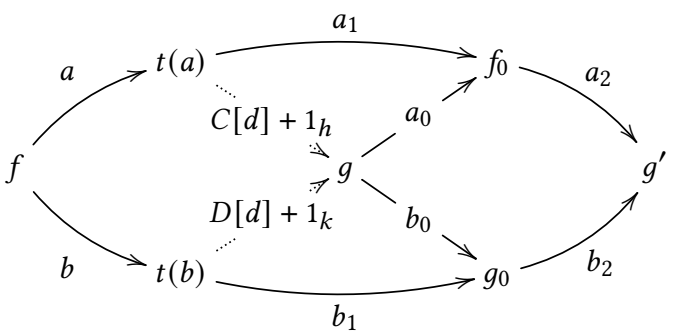

Let us consider the top half of the diagram. Writing the rewriting path $c$ as $c_{1} \cdot \ldots \cdot c_{p}$ and the context $C$ as $\sum_{j=1}^{q} \lambda_{j} C_{j}$, the idea is to apply each $c_{i}$ to each $C_{j}$, leaving the other monomials unchanged: for $1 \leqslant i \leqslant p$ and $1 \leqslant j \leqslant q$, we choose the edge in $\mathcal{R}_{X}$

$$
c_{i, j}:=\sum_{\ell=1}^{j-1} \lambda_{\ell} C_{\ell}\left[t\left(c_{i}\right)\right]+\lambda_{j} C_{j}\left[c_{i}\right]+\sum_{\ell=j+1}^{q} \lambda_{\ell} C_{\ell}\left[s\left(c_{j}\right)\right]+1_{h},
$$

and write $g_{i, j}:=t\left(c_{i, j}\right)$ and $g_{i, 0}:=s\left(c_{i, 1}\right)=t\left(c_{i-1, q}\right)$. Then we have the path in $\mathcal{R}_{X}$

$$
\begin{aligned}
t(a)= & g_{1,0} \stackrel{c_{1,2}}{\longrightarrow} \ldots \stackrel{c_{1, q}}{\longrightarrow} g_{1, q}=g_{2,0} \stackrel{c_{2,1}}{\longrightarrow} \ldots \\
& \ldots \stackrel{c_{p-1, q}}{\longrightarrow} g_{p-1, q}=g_{p, 0} \stackrel{c_{p, 1}}{\longrightarrow} \ldots \stackrel{c_{p, q}}{\longrightarrow} g_{p, q}=g .
\end{aligned}
$$

Moreover, for every $i, j, g_{i, j} \prec_{R} f$, so $X$ is confluent at $g_{i, j}$. Following the second point of Lemma 3.1, there exist rewriting paths $a_{0}, a_{1}$ as in the diagram. Similarly, we show the existence of the rewriting paths $b_{0}, b_{1}$. Since $g \prec_{R} f$, we can apply the confluence hypothesis to the branching $\left(a_{0}, b_{0}\right)$, so we get the rewriting paths $a_{2}, b_{2}$.

TheOREM 3.3. Let $X$ be a terminating ORS. If every essential $S$ polynomial reduces to 0 , then every $S$-polynomial reduces to 0 .

Proof. It suffices to prove that every branching of $X$ is confluent. Since every essential $S$-polynomial reduces to 0 , every essential branching is confluent.

We proceed by well-founded induction on the sources of the branchings of $X$, with the order $<_{R}$, to prove that $X$ is confluent at every polynomial of $\mathcal{F}(\Sigma)$. A reduced polynomial cannot be the source of a local branching, so $X$ is confluent at every $f \in \operatorname{NF}(X)$. Now, fix a nonreduced polynomial $f_{0}$ of $\mathcal{F}(\Sigma)$, and assume that $X$ is confluent at every $g \prec_{R} f_{0}$. Then we proceed by case analysis on the types of local branchings.

Additive branchings. Let $\left(\lambda a+\mu 1_{v}+1_{h}, \lambda 1_{u}+\mu b+1_{h}\right)$ be an additive branching of source $f_{0}$, where $a: u \rightarrow f, b: v \rightarrow g \in \mathcal{R}_{X}^{m}$, $\lambda, \mu \in \mathbf{k} \backslash\{0\}$, and $h \in \mathcal{F}(\Sigma)$, with $u \neq v$ and $u, v \notin \operatorname{Supp}(h)$. We have the following diagram:

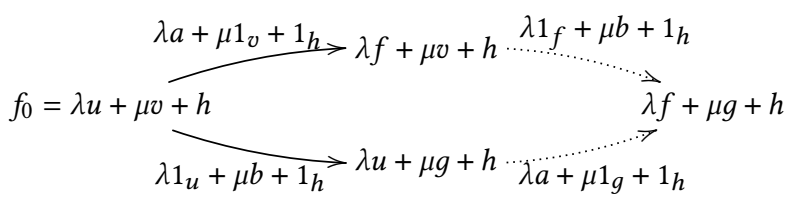

The dotted arrows are rewriting paths in context, so by Lemma 3.2, this local branching is confluent. 
Multiplicative branchings. Let $\left(\lambda C\left[a, 1_{v}\right]+1_{h}, \lambda C\left[1_{u}, b\right]+1_{h}\right)$ be a multiplicative branching of source $f_{0}$, where $C$ is a monomial two-hole context, $a: u \rightarrow f, b: v \rightarrow g \in \mathcal{R}_{X}^{m}, \lambda \in \mathbf{k} \backslash\{0\}, h \in \mathcal{F}(\Sigma)$, and $C[u, v] \notin \operatorname{Supp}(h)$. We have the following diagram:

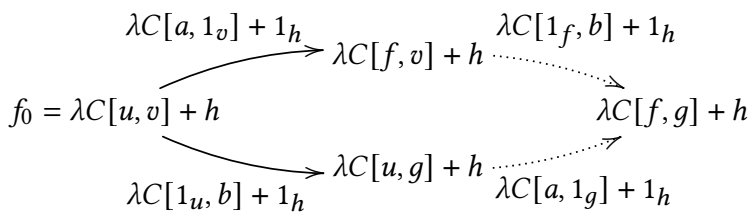

The dotted arrows are rewriting paths in context, so by Lemma 3.2, this local branching is confluent.

Critical branchings. Let $(C[\alpha], D[\beta])$ be a critical branching of source $f_{0}$. If the branching is essential, then it is confluent by hypothesis. Otherwise, there exists a rewriting monomial $E[\gamma]$ such that $C[\alpha] \prec_{\mathrm{rm}} E[\gamma] \prec_{\mathrm{rm}} D[\beta]$, and we get two branchings $(C[\alpha], E[\gamma])$ and $(E[\gamma], D[\beta])$. The branching $(C[\alpha], E[\gamma])$ is either multiplicative or intersecting. If it is multiplicative, then it is confluent by the multiplicative case. Otherwise, it is either non-minimal for $\sqsubseteq$ or a critical branching. In the non-minimal case, there exists a factorisation

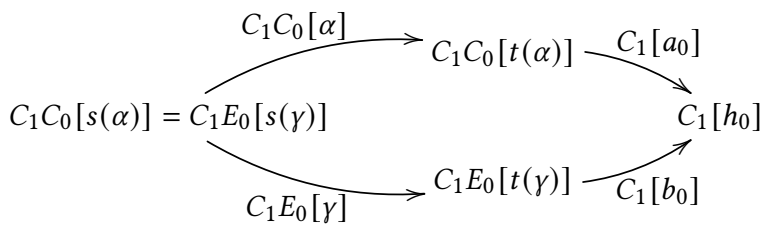

where $\left(C_{0}[\alpha], E_{0}[\gamma]\right)$ is a critical branching and $C_{1}$ is a monomial context. In the critical case, the branching is either essential, or not. If it is essential, then it is confluent by hypothesis. Otherwise, we proceed by induction on the number of rewriting monomials $F[\delta]$ such that $C[\alpha] \prec_{\mathrm{rm}} F[\delta] \prec_{\mathrm{rm}} E[\gamma]$.

We proceed similarly for the branching $(E[\gamma], D[\beta])$ : in every case, we can write

- the branching $(C[\alpha], E[\gamma])$ as $\left(C_{1} C_{0}[\alpha], C_{1} E_{0}[\gamma]\right)$, with $C_{1}$ a monomial context and $\left(C_{0}[\alpha], E_{0}[\gamma]\right)$ a confluent branching,

- the branching $(E[\gamma], D[\beta])$ as $\left(D_{1} E_{0}^{\prime}[\gamma], D_{1} D_{0}[\beta]\right)$, with $D_{1}$ a monomial context and $\left(E_{0}^{\prime}[\gamma], D_{0}[\beta]\right)$ a confluent branching.

We can then construct the confluent diagram

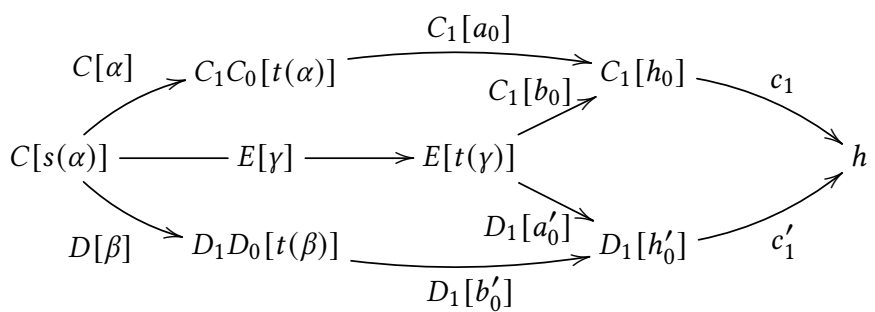

where the left squares are the aforementioned confluent branchings in context and the right square is given by induction hypothesis.
Non-critical intersecting branchings. Finally, let $\left(\lambda C[\alpha]+1_{h}, \lambda D[\beta]+\right.$ $1_{h}$ ) be an intersecting branching of source $f_{0}$ that is not critical, where $\alpha: u \rightarrow f, \beta: v \rightarrow g \in R, C, D$ are monomial contexts, $\lambda \in \mathbf{k} \backslash\{0\}$, and $h \in \mathcal{F}(\Sigma)$, such that $C[u] \notin \operatorname{Supp}(h)$. Let $C_{0}, C_{1}, D_{0}$ be the monomial contexts such that $C_{1} C_{0}=C, C_{1} D_{0}=D$, and $\left(C_{0}[\alpha], D_{0}[\beta]\right)$ is a critical branching. By the previous case, we have the confluence diagram

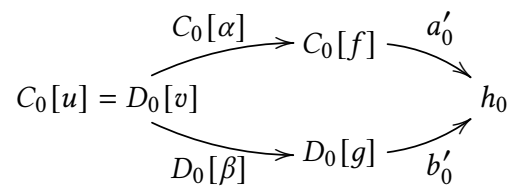

Applying the context $\lambda C_{1}$ and adding $h$, we get the following diagram

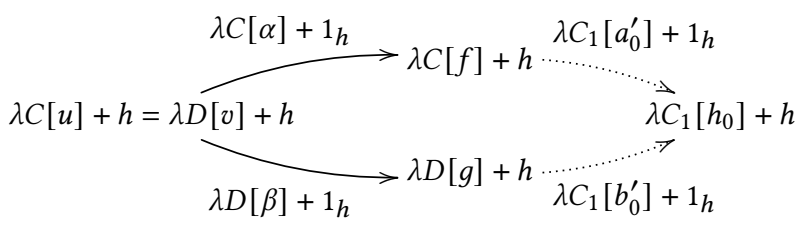

Applying Lemma 3.2, we conclude that the local branching is confluent.

Thus we have shown that the ORS $X$ is locally confluent at $f_{0}$. To conclude the induction step, we show that $X$ is confluent at $f_{0}$ by the diamond lemma. Let $(a, b)$ be a non-local branching of source $f_{0}$, and write $a=a_{0} \cdot a_{1}, b=b_{0} \cdot b_{1}$, where $a_{0}, b_{0}$ are rewriting steps and $a_{1}, b_{1}$ are rewriting paths. We construct the confluence diagram

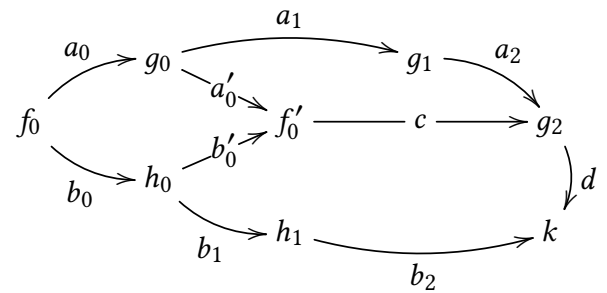

as follows. By the previous arguments, the local branching $\left(a_{0}, b_{0}\right)$ is confluent, yielding the rewriting paths $a_{0}^{\prime}, b_{0}^{\prime}$. Since both $f_{0}>$ $g_{0}$ and $f_{0}>h_{0}$, the induction hypothesis applies to the branching $\left(a_{1}, a_{0}^{\prime}\right)$ to gives the rewriting paths $a_{2}, c$, and then to the branching $\left(b_{0}^{\prime} \cdot c, b_{1}\right)$ to give the rewriting paths $d, b_{2}$.

As a consequence of Theorem 3.3 and [6, Thm. 1], we have the following result.

Corollary 3.4. Let I be an ideal of $\mathcal{F}(\Sigma)$ and $(\Sigma, R)$ a convergent ORS such that $R$ is compatible with a monomial order and $I(R)=I$. If the set of essential S-polynomials reduces to 0 , then $\partial R$ is a Gröbner basis of I.

Completion algorithm. From Theorem 3.3, we deduce a completion algorithm COMPLETE, Algorithm 2, which transforms a terminating ORS into a convergent one presenting the same operad. The algorithm only tests essential $S$-polynomials, using the algorithm ESSENTIALBRANCHINGS defined above. The essential branchings are computed with respect to a monomial order on rewriting monomials $\prec_{\text {rm }}$ given by the function RewritingMonOmialOrder $\left(<^{2},-1\right)$, 
which takes as arguments a monomial order $\prec^{2}$ on $\mathcal{T}(\Sigma)$ and a total order $\dashv$ on $R$, and returns the monomial order on rewriting monomials induced by $\prec^{2},-1$, and the path-lexicographic order on contexts.

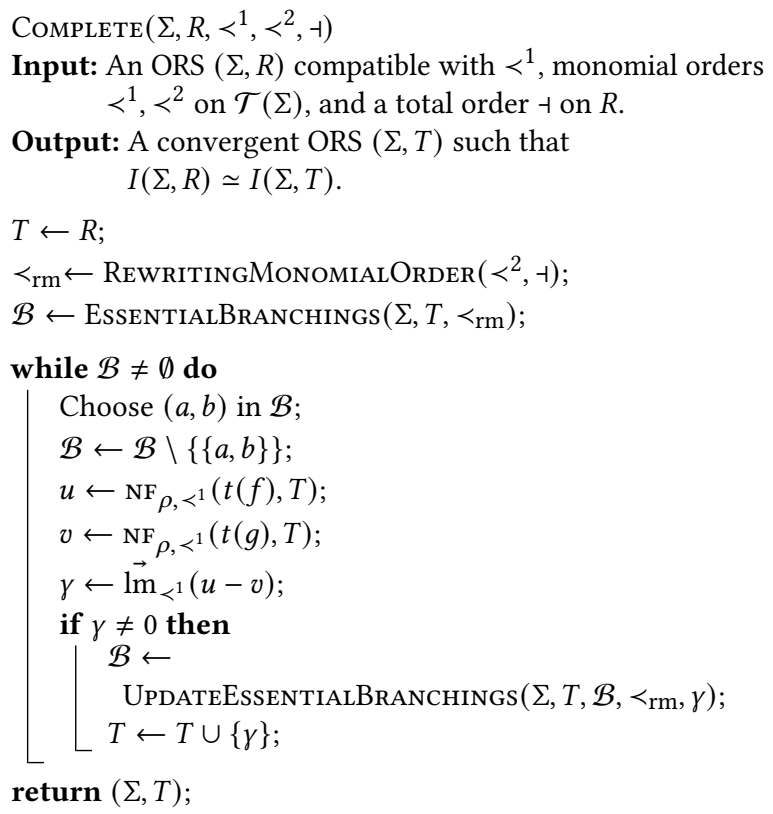

Algorithm 2: The completion algorithm CoMplete

Note that we extend and update during the total order on $R$ to a total order on the rules of $T$. Indeed, the sources of the new rules $\gamma$ never coincide the other rules in $T$. The algorithm Complete requires Algorithm 3, which updates the essential branchings during completion.

Algorithm 2 adds to the set of rules $R$ the rules needed to resolve the essential critical branchings. By construction, the added rules do not change the presented operad. At the output of the algorithm, we get a convergent set of rules $T$. Termination follows from the fact that all added rules are oriented with respect the monomial order $\prec^{1}$. Note that $T$ may be infinite if the completion algorithm loops indefinitely.

This algorithm improves Buchberger's completion algorithm [4] when there are non-essential branchings. However, when the algorithm starts with an ORS whose rules' sources are all of size two and are pairwise distinct, then every branching is essential.

Example. Consider the ORS

$$
\left\langle\begin{array}{l|l}
x_{2}, y_{1}, z_{1} & \begin{array}{l}
\alpha:(x \mid y y 1) \rightarrow(x \mid z z 1), \\
\beta:(x \mid y 1 y) \rightarrow(x \mid z 1 z), \\
\gamma:(x \mid 1 y y) \rightarrow(x \mid 1 z z)
\end{array}
\end{array}\right|
$$

There are three critical branchings: $\left(\alpha \circ_{3} y, \beta \circ_{2} y\right),\left(\alpha \circ_{3} y, \gamma \circ_{1}\right.$ $y),\left(\beta \circ_{2} y, \gamma \circ_{1} y\right)$. The contexts involved, in path-lexicographic order, are $\square_{3} \circ_{3} y \prec \square_{3} \circ_{2} y<\square_{3} \circ_{1} y$. Thus COMPLETE will execute as follows:

- The initial set of essential branchings is $\mathcal{B}=\left\{\left(\alpha \circ_{3} y, \beta \circ_{2}\right.\right.$ $\left.y),\left(\alpha \circ_{3} y, \gamma \circ \circ_{1} y\right)\right\}$.

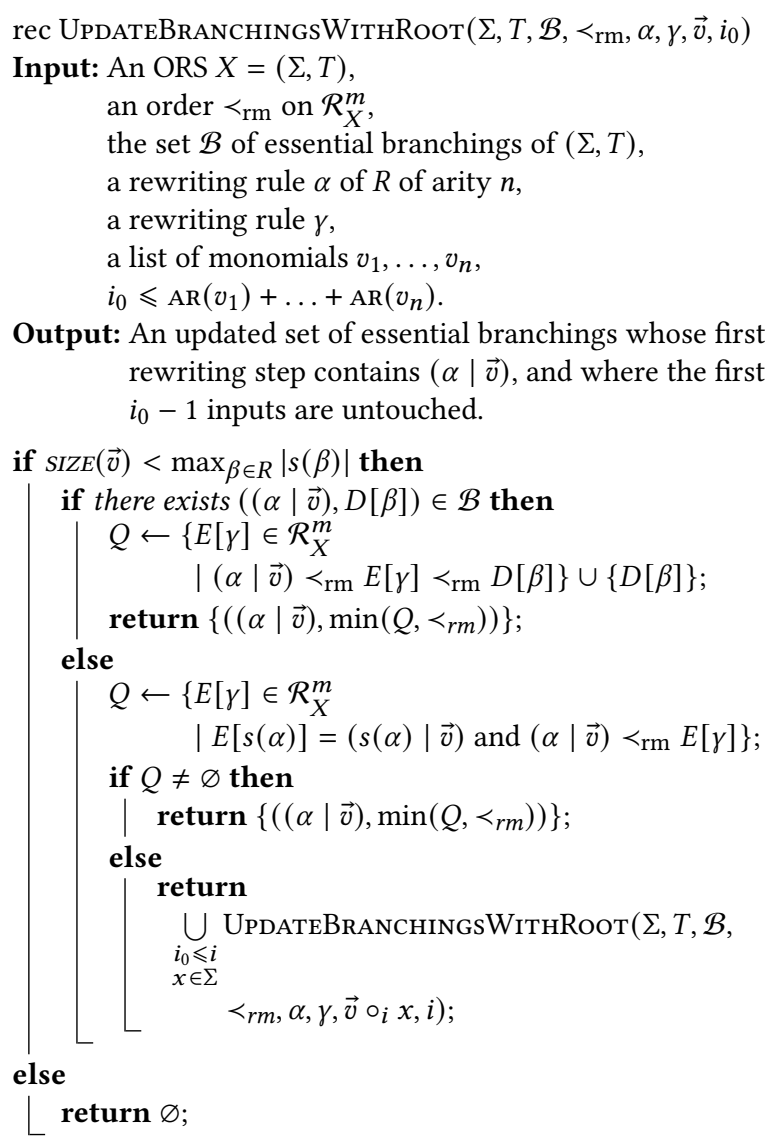

UpdateEssentialBranchings $\left(\Sigma, T, \mathcal{B}, \prec_{\mathrm{rm}}, \gamma\right)$

Input: An ORS $X=(\Sigma, R)$,

A rewriting monomial order $\prec_{\text {rm }}$ on $\mathcal{R}_{X}^{m}$.

Output: The set of essential branchings of $X$.

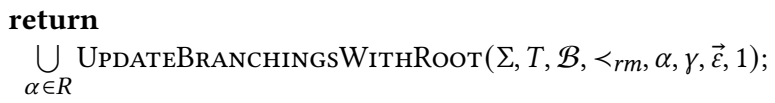

Algorithm 3: UpdateEssentialBranchings and its auxiliary recursive function, UPDATEBRANCHINGSWITHROOT

- Consider the first essential branching $\left(\alpha \circ_{3} y, \beta \circ_{2} y\right.$ ): the targets of these rewriting monomials are already in normal form, so we add $\delta:(x \mid z y z) \rightarrow(x \mid z z y)$.

- No new branchings are created, so $\mathcal{B}$ remains unchanged.

- There remains one essential branching $\left(\alpha \circ_{3} y, \gamma \circ_{1} y\right)$. Similarly, the targets of the two rewriting monomials are already in normal form, so we add $\zeta:(x \mid y z z) \rightarrow(x \mid$ $z z y$ ).

- There are no new essential branchings. $\mathcal{B}$ is empty, so we return the convergent $\mathrm{ORS}(\{x, y, z\},\{\alpha, \beta, \gamma, \delta, \zeta\})$. 


\section{Gröbner bases for syzygies}

In this section, we show that essential and multiplicative syzygies generate the bimodule of syzygies of a convergent ORS $X$. In particular, when the rules of $X$ are compatible with a monomial order, we show how to construct a Gröbner basis of this bimodule with respect a fixed normalization strategy.

Syzygies as loops. Let $X$ be an ORS. A loop of $X$ is a zig-zag $a=a_{1}^{\varepsilon_{1}} \cdot \ldots \cdot a_{n}^{\varepsilon_{n}}$ of rewriting steps, where $a_{i}=\lambda_{i} C_{i}\left[\alpha_{i}\right]+1_{g_{i}}$ and $\varepsilon_{i}= \pm 1$, such that $s\left(a_{1}^{\varepsilon_{1}}\right)=t\left(a_{n}^{\varepsilon_{n}}\right)$. To such a loop, we associate the syzygy $\sum_{i=1}^{n} \varepsilon_{i} \lambda_{i} C_{i}\left[\alpha_{i}\right]$. Conversely, for a syzygy $\sum_{i=1}^{n} \lambda_{i} C_{i}\left[\alpha_{i}\right]$, consider the following path in the graph $\mathcal{R}_{X}$ :

$$
\begin{aligned}
a= & \left(\lambda_{1} \alpha_{1}+\lambda_{2} C\left[s\left(\alpha_{2}\right)\right]+\ldots+\lambda_{n} C\left[s\left(\alpha_{n}\right)\right]\right) \\
& \cdot\left(\lambda_{1} C\left[t\left(\alpha_{1}\right)\right]+\lambda_{2} \alpha_{2}+\ldots+\lambda_{n} C\left[s\left(\alpha_{n}\right)\right]\right) \\
& \ldots \quad \cdot\left(\lambda_{1} C\left[t\left(\alpha_{1}\right)\right]+\lambda_{2} C\left[t\left(\alpha_{2}\right)\right]+\ldots+\lambda_{n} \alpha_{n}\right)
\end{aligned}
$$

We have $s(a)=t(a)=C\left[s\left(\alpha_{1}\right)\right]+\ldots+C\left[s\left(\alpha_{n}\right)\right]$. By the first point of Lemma 3.1, there exists a zig-zag sequence of rewriting steps that loops on $s(a)=t(a)$. In particular, a confluence diagram can be seen as a loop, and so it is associated to a syzygy.

Given a normalization strategy $\rho$, for each essential branching $(f, g)$, let $\left(f \cdot \rho_{f}, g \cdot \rho_{g}\right)$ be the confluence given by $\rho$, and take the corresponding essential syzygy. For each multiplicative branching $(C[\alpha, s(\beta)], C[s(\alpha), \beta])$, take the multiplicative syzygy $C[\alpha, \partial(\beta)]-C[\partial(\alpha), \beta]$. Denote by $\operatorname{Syz}(X, \rho)$ the set of essential and multiplicative syzygies. This notion of syzygy allows us to reformulate Theorem 3.3 as follows:

Proposition 4.1. Let $X$ be a terminating ORS and let $\rho$ be a normalisation strategy. For every branching of $X$, there is a confluence diagram such that the associated syzygy is generated by $\operatorname{Syz}(X, \rho)$.

The proof of this result is obtained following the arguments of the proof of Theorem 3.3 and keeping track of the syzygies corresponding to each confluent diagram.

Proposition 4.2. Let $X$ be a convergent ORS and $\rho$ a normalization strategy. The set $\operatorname{Syz}(X, \rho)$ generates the $\mathcal{F}(X)$-bimodule of syzygies $\mathcal{S}(X)$.

Proof. Let $a$ be a rewriting step in $\mathcal{R}_{X}^{+}$. By Proposition 4.1, the associated syzygy of the confluence diagram of the branching $\left(\rho_{f_{1}}, a \cdot \rho_{f_{2}}\right)$ :

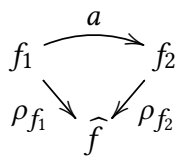

is generated by $\operatorname{Syz}(X, \rho)$. Now consider a syzygy $\mathfrak{s}$ of $X$. As shown above, it corresponds to $\sum_{i=1}^{n} \varepsilon_{i} \partial\left(a_{i}\right)$, where $a_{i} \in \mathcal{R}_{X}^{+}$and $\varepsilon_{i}= \pm 1$ such that $a_{1}^{\varepsilon} \cdot a_{2}^{\varepsilon} \cdot \ldots \cdot a_{n}^{\varepsilon}$ is a loop in $\mathcal{G}_{X}$. This loop can be drawn as a circular pie

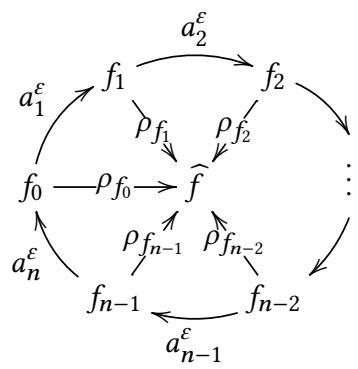

where $\widehat{f}$ is the unique normal form of the polynomials $f_{i}$. Each 'slice' of the pie defines a syzygy generated by $\operatorname{Syz}(X, \rho)$. Combining these syzygies, we conclude that the syzygy $\mathfrak{s}$ is generated by $\operatorname{Syz}(X, \rho)$.

Considering the operad presented by $X$, we compute syzygies modulo the multiplicative syzygies, and we get the following result.

Corollary 4.3. Let $X$ be a convergent ORS, $\rho$ a normalisation strateg $y$, and $\bar{X}$ the operad presented by $X$. The set of essential syzygies generates the $\bar{X}$-bimodule of syzygies $\overline{\mathcal{S}}(X)$.

Let us prove the main result of this section, which corresponds to a non-commutative version of Schreyer's syzygy theorem [21], already apparent in Janet's approach [13].

THEOREM 4.4. Let $X$ be a convergent ORS compatible with a monomial order $<$ and $\rho$ a normalisation strategy. The set $\operatorname{Syz}(X, \rho)$ forms a Gröbner basis of the $\mathcal{F}(X)$-bimodule $\mathcal{S}(X)$ with respect to $<_{r m}$.

Proof. Let $\mathfrak{s}=\sum_{i=1}^{n} \lambda_{i} C_{i}\left[\alpha_{i}\right]$ be a syzygy in $\mathcal{S}(X)$, where $\lambda_{i} \in$ $\mathbf{k} \backslash\{0\}, C_{i} \in \mathcal{R}_{X}^{m}$ and $\alpha_{i} \in R$. Reduce $\mathfrak{s}$ to a normal form wrt $\operatorname{Syz}(X, \rho)$ and the order $\prec_{\mathrm{rm}}$, which we write $\mathrm{t}=\sum_{j=1}^{p} \mu_{j} D_{j}\left[\beta_{j}\right]$, with $\mu_{j} \in \mathbf{k} \backslash\{0\}, D_{j} \in \mathcal{R}_{X}^{m}$ and $\alpha_{j} \in R$. To show that $\operatorname{Syz}(X, \rho)$ forms a Gröbner basis of $\mathcal{S}(X)$, it suffices to show $\mathrm{t}=0$.

For all $1 \leqslant j<j^{\prime} \leqslant p, D_{j}\left[s\left(\beta_{j}\right)\right] \neq D_{j^{\prime}}\left[s\left(\beta_{j^{\prime}}\right)\right]$. Otherwise, this would give a local branching of the form $\left(D_{j}\left[\beta_{j}\right], D_{j^{\prime}}\left[\beta_{j^{\prime}}\right]\right)$. By convergence of $X$, this would give a syzygy whose leading term is either $D_{j}\left[\beta_{j}\right]$ or $D_{j^{\prime}}\left[\beta_{j^{\prime}}\right]$. By Proposition 4.2 , the set $\operatorname{Syz}(X, \rho)$ generates all syzygies, so in particular, the previous syzygy could be reduced by an element of $\operatorname{Syz}(X, \rho)$. This would contradict the fact that $\mathrm{t}$ is a normal form.

As a consequence, the monomials $D_{j}\left[s\left(\beta_{j}\right)\right]$ in $\mathrm{t}$ cannot cancel each other out. Moreover, by definition of syzygies,

$$
\sum_{j=1}^{p} \mu_{j} D_{j}\left[\partial\left(\beta_{j}\right)\right]=\sum_{i=1}^{n} \lambda_{i} C_{i}\left[\partial\left(\alpha_{i}\right)\right]=0 .
$$

The only possibility is $p=0$, so $t=0$.

\section{Further developments}

In this article, we have introduced a notion of rewriting systems for non-symmetric operads whose rules do not depend on a monomial order. We have shown how to optimize a completion procedure that given a terminating ORS produces a convergent one by resolving only essential $S$-polynomials. We also have proved how to compute a Gröbner basis of the bimodule of syzygies using essential syzygies. Several improvements of these constructions and results are 
possible. In particular, some variations are possible on the notion of essential $S$-polynomials. Moreover, we can extend our completion algorithm to algebras over operads and shuffle operads.

Essential branchings: continuation. One of the objectives of the rewriting approach developed in this work is to compute explicit free resolutions, and more generally cofibrant replacements for associative algebras $[1,2,11]$ and operads $[7,17]$. In this direction, it is necessary to extend the definition of essential branchings to higher dimensions. To do so, we consider an alternate definition of essential branchings based on the notion of crowns.

Let $X=(\Sigma, R)$ be an ORS. A reducible divisor of $u \in \mathcal{T}(\Sigma)$ is a minimal non-reduced monomial $v$ such that $v \subseteq u$. Denote by $D(u)$ the set of reducible divisors of $u$. When the composition $(u \mid \vec{v})$ is defined, we say that $\vec{v}$

i) creates reducible divisors if $D(u \mid \vec{v}) \backslash D(u)$ is nonempty,

ii) creates reducible divisors context-minimally if, in addition, for all submonomials $w_{i}$ of $v_{i}$ and monomials $\vec{w}^{\prime} \neq \vec{\varepsilon}$ such that $(u \mid \vec{v})=\left((u \mid \vec{w}) \mid \vec{w}^{\prime}\right), D(u \mid \vec{w})=D(u)$,

iii) is a crown on $u$ if the monomials in $\vec{v}$ are reduced and create reducible divisors context-minimally.

By definition, essential branchings correspond to critical branchings of the form $((\alpha \mid \vec{v}), D[\beta])$ where $\vec{v}$ is a crown on $s(\alpha)$. In this way, essential branchings can be generalized to a notion of essential overlappings involving $n$ rewriting rules as done in [17], where the authors show that higher dimensional essential overlappings generate higher dimensional syzygies on the presented operad $\bar{X}$, providing a resolution of the operad presented by the ORS $X$, and thus extending Corollary 4.3 to higher dimensions.

Algebras over operads. Our approach can be adapted to rewriting systems for algebras over non-symmetric operads. Indeed, an algebra $A$ over a non-symmetric operad $P$ can be represented by the polynomials of arity 0 of an operad whose generators are those of $P$ and $A$, the latter being of arity 0 , satisfying the relations of the operad and those of the algebra [8]. Gröbner bases have been developed for algebras over certain operads, including the commutative operad and associative operad for usual algebras, as well as the Lie operad [5] and the free operad on a binary generator, which defines non-associative non-commutative algebras [10]. We expect that our approach is a first step towards a unified definition of critical branchings for rewriting systems in algebraic structures.

The case of shuffle operads. In this article, for simplicity's sake, our algorithms are written for non-symmetric operads. However, it would work for other types of operads, such as shuffle operads and colored versions of non-symmetric and shuffle operads. Shuffle operads, which encode symmetric actions explicitly, were introduced by Dotsenko and Khoroshkin in [6] to study homological properties of linear symmetric operads by a rewriting approach. They introduced a notion of Gröbner bases for shuffle operads with respect to a total order on tree monomials and a completion algorithm that extends Buchberger's completion algorithm.

A free shuffle operad consists of the same monomials as a nonsymmetric operad, except that we allow certain permutations of inputs: a shuffle monomial is of the form

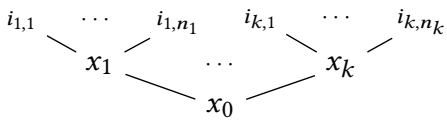

where the inputs are permuted such that $i_{j, 1}<\cdots<i_{j, n_{j}}$ for all $1 \leqslant j \leqslant k$ and $i_{1,1}<\cdots<i_{k, 1}$. Our constructions and algorithms on non-symmetric operads can be extended to shuffle operads: in particular, we have an analogous notion of essential branchings. The key modification consists in the definition of the set of monomials $\mathcal{T}(\Sigma)$ by taking into account the shuffle composition: $\left(\left.x_{0}\right|_{f} x_{1} \ldots x_{k}\right)$, where $f$ is the surjection associated to the permutation on the inputs. The shuffle composition extends to contexts and the confluence properties work as well. Theorems 3.3 and 4.4 still apply, and the algorithms EssentialBranchings and COMPLETE can be adapted to the shuffle case.

\section{References}

[1] David J. Anick. On the homology of associative algebras. Trans. Amer. Math. Soc., 296(2):641-659, 1986.

[2] David J. Anick and Edward L. Green. On the homology of quotients of path algebras. Comm. Algebra, 15(1-2):309-341, 1987.

[3] Murray R. Bremner and Vladimir Dotsenko. Algebraic operads. CRC Press, Boca Raton, FL, 2016. An algorithmic companion.

[4] Bruno Buchberger. An Algorithm for Finding the Basis Elements in the Residue Class Ring Modulo a Zero Dimensional Polynomial Ideal. PhD thesis, University of Innsbruck, 1965. J. Symbolic Comput. Vol. 41 (3-4): 475-511, 2006.

[5] W. A. de Graaf and J. Wisliceny. Constructing bases of finitely presented lie algebras using gröbner bases in free algebras. In Proceedings of the 1999 International Symposium on Symbolic and Algebraic Computation, ISSAC '99, page 37-43, New York, NY, USA, 1999. Association for Computing Machinery.

[6] Vladimir Dotsenko and Anton Khoroshkin. Gröbner bases for operads. Duke Math. J., 153(2):363-396, 2010.

[7] Vladimir Dotsenko and Anton Khoroshkin. Quillen homology for operads via Gröbner bases. Doc. Math., 18:707-747, 2013.

[8] Vladimir Dotsenko and Bruno Vallette. Higher Koszul duality for associative algebras. Glasg. Math. f., 55(A):55-74, 2013.

[9] Rüdiger Gebauer and H. Michael Möller. On an installation of Buchberger's algorithm. F. Symbolic Comput., 6(2-3):275-286, 1988. Computational aspects of commutative algebra.

[10] Lothar Gerritzen. Tree polynomials and non-associative Gröbner bases. F. Symbolic Comput., 41(3-4):297-316, 2006.

[11] Yves Guiraud, Eric Hoffbeck, and Philippe Malbos. Convergent presentations and polygraphic resolutions of associative algebras. Math. Z., 293(1-2):113-179, 2019.

[12] Eric Hoffbeck. A Poincaré-Birkhoff-Witt criterion for Koszul operads. Manuscripta Math., 131(1-2):87-110, 2010.

[13] Maurice Janet. Sur les systèmes d'équations aux dérivées partielles. Fournal de mathématiques pures et appliquées, 8(3):65-151, 1920.

[14] Donald Knuth and Peter Bendix. Simple word problems in universal algebras. In Computational Problems in Abstract Algebra (Proc. Conf., Oxford, 1967), pages 263-297. Pergamon, Oxford, 1970.

[15] Viktor Levandovskyy, Tobias Metzlaff, and Karim Abou Zeid. Computation of free non-commutative Gröbner Bases over Z with Singular:Letterplace. In ISSAC 2020 - International Symposium on Symbolic and Algebraic Computation, Kalamata, Greece, July 2020.

[16] Jean-Louis Loday. La renaissance des opérades. Astérisque, 237:Exp. No. 792, 3 , 47-74, 1996. Séminaire Bourbaki, Vol. 1994/95.

[17] Philippe Malbos and Isaac Ren. Shuffle polygraphic resolutions for operads. preprint, arXiv:2012.15718, December 2020.

[18] J. P. May. The geometry of iterated loop spaces. Springer-Verlag, Berlin-New York, 1972. Lectures Notes in Mathematics, Vol. 271.

[19] Teo Mora. An introduction to commutative and noncommutative Gröbner bases. Theoret. Comput. Sci., 134(1):131-173, 1994. Second International Colloquium on Words, Languages and Combinatorics (Kyoto, 1992).

[20] Maurice Nivat. Congruences parfaites et quasi-parfaites. In Séminaire P. Dubreil, 25e année (1971/72), Algèbre, Fasc. 1, Exp. No. 7, page 9. Secrétariat Mathématique, Paris, 1973.

[21] Frank-Olaf Schreyer. Die berechnung von syzygien mit dem verallgemeinerten weierstrass'schen divisionssatz. Diplom Thesis, University of Hamburg, Germany, 1980, 1980. 